\title{
Assessing cortico-hippocampal functional connectivity under anesthesia and kainic acid using generalized partial directed coherence
}

\author{
Jiannis Taxidis - Ben Coomber • Rob Mason • \\ Markus Owen
}

Received: 18 June 2009 / Accepted: 8 February 2010 / Published online: 4 March 2010

(C) The Author(s) 2010. This article is published with open access at Springerlink.com

\begin{abstract}
A significant challenge in modern neuroscience lies in determining the functional connectivity between discrete populations of neurones and brain regions. In this study, a variation of partial directed coherence, the generalized partial directed coherence (gPDC), along with a newly proposed critical value for $\mathrm{gPDC}$, were applied on recorded local field potentials (LFPs) and single-unit activity, in order to assess information flow between medial prefrontal cortex (mPFC) and hippocampus and within the hippocampus of the rat brain, under isoflurane anesthesia and kainic acid-induced enhanced neuronal activity. Our findings suggest that, under anesthesia, there exists a continuous information flow from hippocampus towards $\mathrm{mPFC}$, reversed mostly during activity bursts occurring in the mPFC. Moreover, there was a clear directional connection from the lateral towards medial dorsal hippocampus, most prominent in the beta frequency band $(10-30 \mathrm{~Hz})$. Kainic acid resulted in partially disrupting the reciprocal cortico-hippocampal connectivity and reversing the intra-hippocampal one. The biological implications of these findings on the effects of anesthesia and kainic acid in brain connectivity, along with implementation issues of gPDC analysis on field potentials and spike trains, are extensively discussed.
\end{abstract}

Keywords Partial directed coherence - Generalized partial directed coherence · Granger causality · Hippocampus .

\footnotetext{
J. Taxidis $(\bowtie) \cdot$ M. Owen

School of Mathematical Sciences, University of Nottingham,

Nottingham, NG7 2RD, UK

e-mail:pmxit@nottingham.ac.uk

B. Coomber · R. Mason

School of Biomedical Sciences, Queen's Medical Centre, University of Nottingham, Nottingham, NG7 2UH, UK
}

Medial prefrontal cortex $\cdot$ Local field potentials . Spike trains

\section{Introduction}

Traditionally effective connectivity between pairs of neuronal structures has been estimated using cross-correlation analysis which gives an indication of a delay in coupling, but does not provide any inference to causality, i.e., which structure drives which. Use of coherence provides a frequency-domain representation of the magnitude and phase relationship between two local neuronal populations, but provides no information on the directionality between them. However, some directionality information can be obtained from the coherence phase spectrum which provides a measure of phase (time delay) between coherent signals (Halliday et al. 1995) but it has been argued to be rather ineffective (Kus et al. 2004).

New techniques for statistical analysis of signals have recently been developed in order to provide tools for assessing the directional effective connectivity between neurons, neural ensembles, and whole brain regions. Among them, the closely related partial directed coherence (PDC) (Sameshima and Baccalá 1999; Baccalá and Sameshima 2001) and Directed Transfer Function (DTF) (Kamiński and Blinowska 1991; Kamiński et al. 2001) have been applied in numerous studies of either simplified tests (Baccalá and Sameshima 2001; Kus et al. 2004; Winterhalder et al. 2005) or real neurophysiological data (reviewed by Pereda et al. 2005). A variation of PDC, the generalized PDC, was also recently introduced to cope with time series with widely different variances (Baccala et al. 2007). These techniques are mainly based on the notion of Granger causality (Granger 1980) and have the advantage that they can be applied on multivariate 
time series, not restricting the connectivity analysis to pairs of neural structures but extending it to whole sets of simultaneously recorded signals. This way, typical problems with pairwise analysis, namely serial connections or common sources (Kus et al. 2004; Cadotte et al. 2008) can be avoided.

In this study, PDC analysis was applied on electrophysiologically recorded local field potentials (LFPs) and unit spike trains from dorsal hippocampus (medial and lateral regions) and medial prefrontal cortex (mPFC) from isoflurane-anesthetised rats (Coomber et al. 2008). After a period of basal recordings, kainic acid (KA) was administered in order to induce increased excitatory activity. The aims of the study were (i) to assess the rat mPFC-hippocampal and intra-hippocampal functional connectivity, under isoflurane anesthesia; (ii) to examine potential changes in neuronal dynamics following KA induction; (iii) to compare the connectivity indicated by PDC on LFPs with that by PDC on spikes, pre-processed by two different techniques.

The experimental methods and recording procedures are described in Sect. 2. A short introduction to Granger causality and the definitions of PDC and generalized PDC are given in Sect. 3. Spike pre-processing procedures and numerical implementation are described in Sect. 4. All results from our analysis are presented in detail in Sect. 5.

\section{Methods}

\subsection{Animals}

All procedures were carried out in accordance with the animals (Scientific Procedures) Act 1986, UK. Experiments were performed on male Lister hooded rats (University of Nottingham Biomedical Sciences Services Unit in-house colony) weighing $200-350 \mathrm{~g}(n=4)$. Rats were group housed on a $12 \mathrm{~h}: 12 \mathrm{~h}$ light:dark cycle, and food and water were available ad libitum. Rats were anesthetized with a $3.5 \%$ isoflurane $/ \mathrm{O}_{2}: \mathrm{N}_{2} \mathrm{O}(50: 50 \%)$ mixture. The isoflurane level was reduced progressively and maintained at $1.5-2 \%$ throughout the experiment to maintain a constant state of areflexia. Core body temperature was monitored throughout and maintained at $37^{\circ} \mathrm{C}$ using a homeothermic heating pad (Harvard Apparatus Ltd., UK).

\subsection{Surgery}

Rats were placed in a stereotaxic frame, craniotomies were performed over dorsal hippocampus and $\mathrm{mPFC}$, and a 16 micro-wire Teflon-coated stainless steel electrode array (NB Labs, USA) was slowly and progressively lowered into the right dorsal hippocampus $[3.5 \mathrm{~mm}$ posterior and $3.0 \mathrm{~mm}$ lateral to bregma, measured from the centre of the array; 3.0$3.5 \mathrm{~mm}$ ventral to the cortical surface; (Paxinos and Watson
1998)], and an eight micro-wire electrode bundle lowered into the right $\mathrm{mPFC}[3.2 \mathrm{~mm}$ anterior and $0.5 \mathrm{~mm}$ lateral to bregma; $2.0-2.5 \mathrm{~mm}$ ventral to the cortical surface; (Paxinos and Watson 1998)]. Multiple extracellular single-unit activity (filtered at $250 \mathrm{~Hz}-8 \mathrm{kHz}$ ) from all electrodes of the 16-wire array and 8-wire bundle, and simultaneous LFPs, sampled at $1 \mathrm{kHz}$ and filtered at $0.1-170 \mathrm{~Hz}$, were recorded using a Plexon Multichannel Acquisition Processor (MAP) system (Plexon Inc., TX, USA).

\subsection{Recording procedure}

Recordings were made in epochs of 3 min every $10 \mathrm{~min}$, over a total period of $123 \mathrm{~min}$. Rats were administered KA (10 $\mathrm{mg} / \mathrm{kg}$ i.p.; Sigma, UK; $n=4$ ) dissolved in saline, after a $33 \mathrm{~min}$ period of basal recording, to induce increased excitatory activity (Westbrook and Lothman 1983; Kunz and Oliw 2001) with a lag of approximately 20-30 min following i.p. administration. Vehicle control injections did not alter the spike firing or the LFP signal activity (data not shown).

\subsection{Histology}

At the end of each experiment, animals were deeply anesthetised and current $(0.1 \mathrm{~mA}$ for $5-10 \mathrm{~s})$ passed through medial, central and lateral pairs of electrodes in the hippocampus and $\mathrm{mPFC}$, to mark placements. Brains were removed and stored in $4 \%$ paraformaldehyde/4\% potassium ferrocyanide solution for $48 \mathrm{~h}$. Histological sections $(200 \mu \mathrm{m})$ were taken with a vibratome (Camden Instruments, UK) and electrode placements were revealed by the Prussian Blue reaction (Hong et al. 2000). Medial and lateral hippocampal electrode placements were largely confined to the CA3 and CA1 fields, respectively; mPFC electrodes were between layers 3-5 (Fig. 1).

\subsection{Spike sorting}

Data from animals with confirmed hippocampal electrode placements were sorted into individual neuronal singleunits using both automatic and manual sorting techniques in Offline Sorter (Plexon Inc.). Briefly, principle component analysis was used to display the recorded waveforms in threedimensional space. Each electrode was manually checked for artifacts (e.g., $50 \mathrm{~Hz}$ noise). Automatic sorting (T-Dist E-M) methods were then used to separate the waveforms into individual units. The resulting clusters were inspected and the units were considered to be separate only if the cluster borders did not overlap (see Stevenson et al. 2007). 
Fig. 1 Schematic representation of histological verification of dorsal hippocampal (upper left) and mPFC (upper right) recording sites. The most medial (black dots) and lateral (white dots) electrode placements of the hippocampal array and an electrode from the bundle in mPFC (black dots) are shown in coronal brain images (Paxinos and Watson 1998) for each experiment included in this study ( $n=4$ rats). The distance posterior to bregma in the sequence of images for hippocampal placements is: (a) $-3.8 \mathrm{~mm}$; (b) $-3.6 \mathrm{~mm}$; (c) $-3.3 \mathrm{~mm}$. The distance anterior to bregma in each image for mPFC placements is: $(a)$ $3.2 \mathrm{~mm}$; (b) $3.7 \mathrm{~mm}$. The lower panel shows representative segments of LFP activity during basal conditions in one rat recorded simultaneously in mPFC, medial hippocampus (mHipp), and lateral hippocampus (lHipp) respectively over a $10 \mathrm{~s}$ period
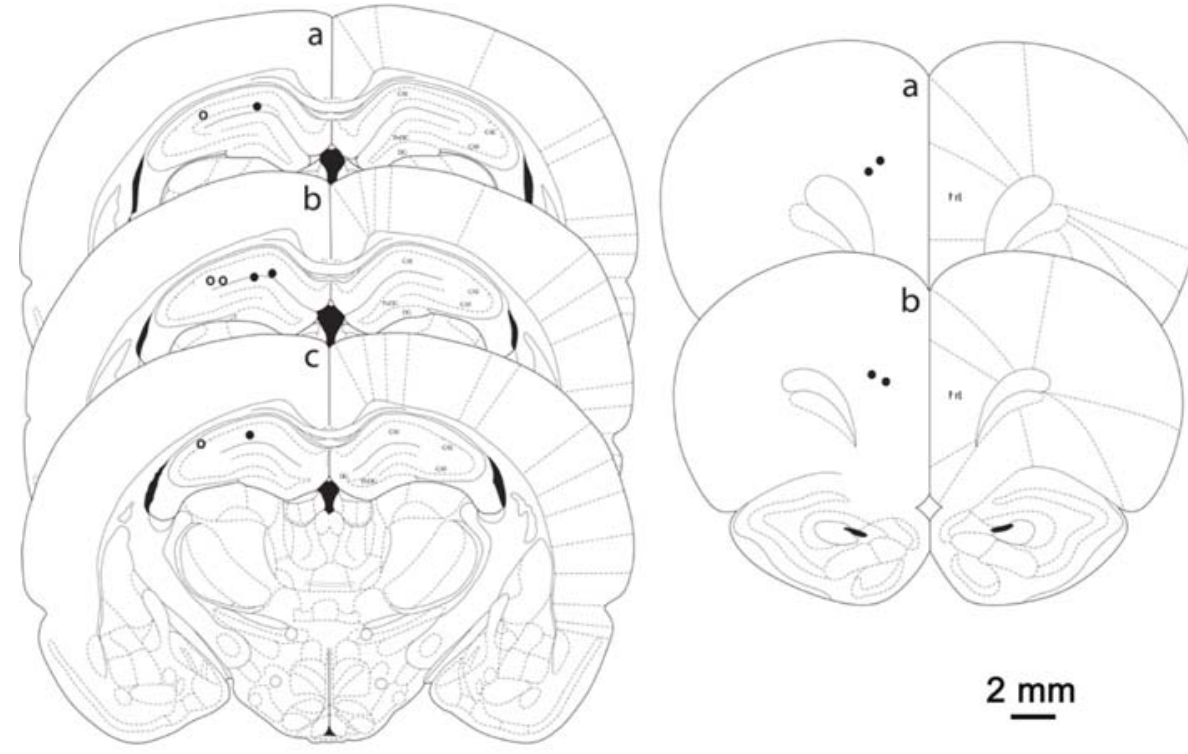

$2 \underline{\mathrm{mm}}$

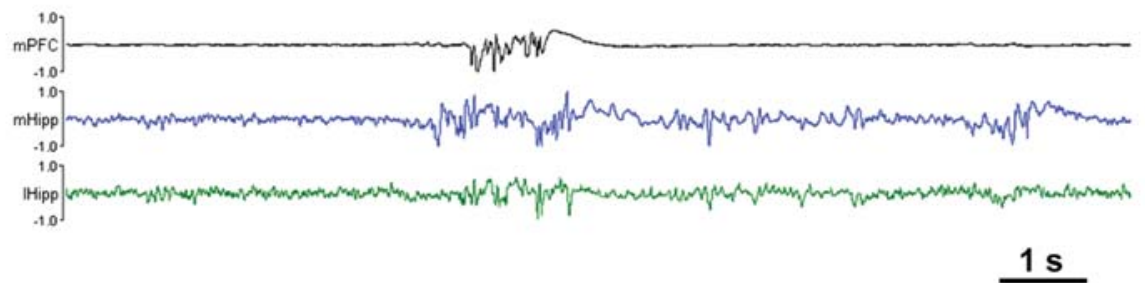

\subsection{Spike selection}

The mean firing rate of each unit data set was computed and only units with firing rates within the range $0.1-10 \mathrm{~Hz}$ in hippocampus and $0.05-10 \mathrm{~Hz}$ in $\mathrm{mPFC}$ were used for the subsequent analysis. This range was assumed to represent populations of putative principal cells (Frank et al. 2001). In order to make the comparison between analysis on the single unit and LFP activity as rigorous as possible, only units recorded adjacent to the electrodes recording LFPs were used. If none of these satisfied the firing rate criteria, then the range of potential units was expanded by one electrode further from the LFP electrode, until there was at least one unit for each brain region.

\section{Partial directed coherence}

The definition of PDC is based on the notion of linear Granger causality (Granger 1980) whose basic concept is simply that the cause precedes its effect temporally. Thus, a variable $x_{j}(t)$ "Granger-causes" another $x_{i}(t)$, if knowledge of $x_{j}(t)$ 's past values significantly improves predictions of $x_{i}(t)$ 's future values. This relation is not reciprocal, meaning that $x_{j}(t)$ can Granger-cause $x_{i}(t)$, without $x_{i}(t)$ necessarily causing $x_{j}(t)$ back, and this lack of reciprocity permits gauging the direc- tion of information flow between variables. Note here that the term "information flow" does not correspond to actual bits of information in the strict sense but is a widely used term in similar studies which implies a directed causal link.

Granger causality within an $m$-variate process defined by a set $\left(x_{1}(t), x_{2}(t), \ldots, x_{m}(t)\right)^{T}$ of $m$ zero-mean stationary time series, i.e., trend-free time series with approximately constant mean and variance, is assessed by modeling them through a vector autoregressive (VAR) model of the form:

$$
\left[\begin{array}{c}
x_{1}(t) \\
\vdots \\
x_{m}(t)
\end{array}\right]=\sum_{r=1}^{\mathrm{p}} A_{\mathrm{r}}\left[\begin{array}{c}
x_{1}(t-r) \\
\vdots \\
x_{m}(t-r)
\end{array}\right]+\left[\begin{array}{c}
u_{1}(t) \\
\vdots \\
u_{m}(t)
\end{array}\right]
$$

where $\left(u_{1}(t), \ldots, u_{m}(t)\right)^{T}$ are uncorrelated Gaussian white noise processes representing the model residuals (Lütkepohl 2005), with covariance matrix $\Sigma . p$ is the VAR model's order which represents the maximum temporal delay in the causal link between the modeled variables. For a VAR process of unknown order, as in this case, it can be optimized through various selection criteria, such as the Akaike criterion (Akaike 1974) or the Bayesian Information Criterion (Schwarz 1978), which acquire their minimum value at the model order which strikes the best balance between the model fit and overparametrization. The stationarity of the series generated by the VAR model can be assessed through the model's 
stability. A VAR model is stable if it satisfies the criterion:

$\operatorname{det}\left(I-A_{1} z-\cdots-A_{\mathrm{p}} z^{\mathrm{p}}\right) \neq 0$

for all $z \in \mathbb{C}$, such that $|z| \leq 1$. The VAR coefficients $A_{\mathrm{r}}$ are $m \times m$ matrices whose entries $A_{\mathrm{r}}(i j)$ represent the linear effect of $x_{j}$ 's past value $x_{j}(t-r)$ onto $x_{i}$ 's present: $x_{i}(t)$. Consequently, if it can be shown that $A_{\mathrm{r}}(i j)$, is significantly nonzero for any $r \in 1,2, \ldots, p$ then $x_{j}$ is Grangercausing $x_{i}$.

Taking the Fourier Transformation of the VAR coefficients:

$A(f)=\sum_{r=1}^{\mathrm{p}} A_{\mathrm{r}} e^{-2 \pi i f r}$

yields a frequency-domain representation of the VAR model. Defining the matrix: $\bar{A}(f)$ as the difference between the identity matrix and $A(f): \bar{A}(f)=I-A(f)$, then PDC from variable $x_{j}$ to $x_{i}$ is defined as:

$\left|\pi_{i \leftarrow j}(f)\right|=\frac{\left|\bar{A}_{i j}(f)\right|}{\sqrt{\sum_{k}\left|\bar{A}_{k j}(f)\right|^{2}}}$

This definition is directly related to Granger causality since $\pi_{i \leftarrow j}$ is zero for all frequencies if and only if $A_{\mathrm{r}}(i j)=0$ for all $r \in 1,2, \ldots, p$, in which case $x_{j}$ is not Grangercausing $x_{i}$

The denominator in (4) is a normalization that bounds the PDC coefficients to values from 0 to 1 . This choice of scaling means that $\left|\pi_{i \leftarrow j}(f)\right|$ measures the outflow of information from signal $x_{j}$ to signal $x_{i}$ with respect to the total outflow of information from $x_{j}$ to all signals. In other words PDC ranks the interaction strength with respect to the origin of the information flow. This is a characteristic difference from the similar measure of DTF (Kamiński and Blinowska 1991), where the interaction is ranked with respect to the total inflow of information to a variable.

The asymptotic distributions of PDC have been recently studied and it was shown that under the null hypothesis of $\left|\bar{A}_{i j}(f)\right|^{2}=0$, equivalent to $\left|\pi_{i \leftarrow j}(f)\right|=0$, the asymptotic distribution of:

$\frac{N}{C_{i j}(f)}\left|\tilde{A}_{i j}(f)\right|^{2}$

is a weighted average of two independent $\chi^{2}$-distributions with one degree of freedom (Schelter et al. 2006a; Takahashi and Baccalá 2007). Here:

$C_{i j}(f)=\sigma_{i}^{2}\left(\sum_{k, l=1}^{\mathrm{p}} H_{j j}(k-l) \cos (2 \pi(k-l) f)\right)$,

$\sigma_{i}^{2}$ is the variance of $u_{i}$ and $H_{j j}(h)$ is the $j$ th diagonal entry in the $(k-l)$-lag block of $H$, where $H$ is the inverse of the
VAR process's covariance matrix $\Gamma_{X}(0)$ (Lütkepohl 2005):

$\Gamma_{X}=\left(\begin{array}{cccc}\Gamma(0) & \Gamma(1) & \ldots & \Gamma(p-1) \\ \Gamma(-1) & \Gamma(0) & \ldots & \Gamma(p-2) \\ \vdots & \vdots & \ddots & \vdots \\ \Gamma(-p+1) & \Gamma(-p+2) & \ldots & \Gamma(0)\end{array}\right)$

$\tilde{A}_{i j}(f)$ is an estimate of the true VAR-process matrix $\bar{A}_{i j}(f)$, computed from the $N$-length time series through the common normally distributed estimators [maximum likelihood, YuleWalker estimators etc. (Lütkepohl 2005)]. The asymptotic distribution of (5) leads to the approximation of an analytical critical level for the significance of a nonzero $\left|\pi_{i \leftarrow j}(f)\right|$, proposed in Schelter et al. (2006a):

$\sqrt{\frac{\hat{C}_{i j}(f) \chi_{1,1-\alpha}^{2}}{N \sum_{k}\left|\tilde{A}_{k j}(f)\right|^{2}}}$

with $\chi_{1,1-\alpha}^{2}$ the $1-\alpha$ quantile of the $\chi^{2}$-distribution with one degree of freedom and $\hat{C}_{i j}(f)$ an estimate of $C_{i j}(f)$ based on the computation of the covariance matrix $\Gamma_{X}$ by the sample data.

Although PDC has proved to be an accurate tool for the detection of direct connectivities, both in cases of coupled oscillators (Baccalá and Sameshima 2001; Winterhalder et al. 2005; Gourévitch et al. 2006; Schelter et al. 2006a) or simple neuronal models of interconnected neurons (Sameshima and Baccalá 1999; Kamiński et al. 2001; Astolfi et al. 2007), it has been shown that large differences in the variances of the modeled time series can yield distortions in the resulting PDC values (Winterhalder et al. 2005; Baccala et al. 2007). For example, a set of three uncorrelated white noise processes, where two of them have much larger variance than the third, will produce a distorted connectivity profile, since PDC wrongly detects connections from the low-variance process to the other two (Winterhalder et al. 2005). To avoid such distortions deriving from differences in time series scaling, a variation of the original PDC, the so called generalized PDC (gPDC) was introduced (Baccala et al. 2007). In gPDC, the coefficients $\left|\bar{A}_{i j}(f)\right|$ are normalized by the standard deviation of the $u_{i}$ model residuals:

$\left|\pi_{i \leftarrow j}^{\text {gen }}(f)\right|=\frac{\frac{1}{\sigma_{i}}\left|\bar{A}_{i j}(f)\right|}{\sqrt{\sum_{k} \frac{1}{\sigma_{k}^{2}}\left|\bar{A}_{k j}(f)\right|^{2}}}$

In need of a critical level for gPDC, we followed the same analysis as in the derivation of (8). Given that the stationarity of the time series guarantees they have bounded non-zero variances, under the null hypothesis of $\frac{1}{\sigma_{i}^{2}}\left|\bar{A}_{i j}(f)\right|^{2}=0$, 
Fig. 2 PDC (dashed line) and gPDC (solid line) for three independent white noises with different variance (see text). PDC indicates that process $x_{1}$ is driving the much higher variant processes $x_{2}$ and $x_{3}$. gPDC on the other hand, being practically zero and lying entirely below the critical value (11) (dotted line) restores the independence of the processes. The diagonal panels represent the logarithm of the power spectral density of each process
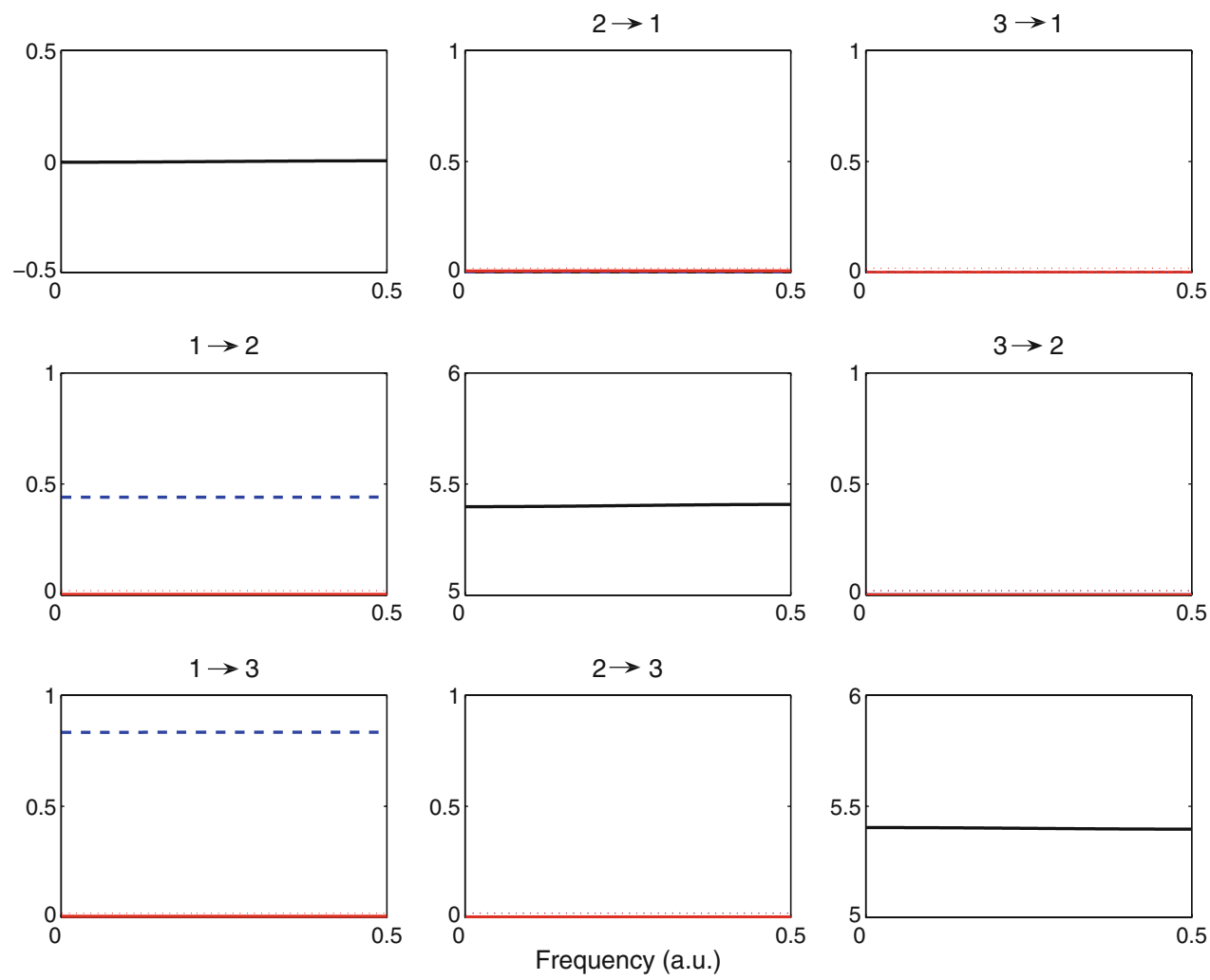

which is equivalent to $\left|\pi_{i \leftarrow j}^{\text {gen }}(f)\right|=0$, we can simply rewrite (5) as:

$$
\frac{N}{\frac{C_{i j}(f)}{\sigma_{i}^{2}}} \frac{1}{\sigma_{i}^{2}}\left|\tilde{A}_{i j}(f)\right|^{2}
$$

whose asymptotic distribution is again a weighted average of two independent $\chi^{2}$-distributions with one degree of freedom. By following same steps as in Schelter et al. (2006a), we obtain a similar approximation to (8) for the significance level of gPDC:

$$
\sqrt{\frac{\hat{C}_{i j}(f) \chi_{1,1-\alpha}^{2}}{N \sigma_{i}^{2} \sum_{k} \frac{1}{\sigma_{k}^{2}}\left|\tilde{A}_{k j}(f)\right|^{2}}}
$$

where $\hat{C}_{i j}(f), \sigma_{i}$ and $\tilde{A}_{i j}(f)$ are estimated as above.

It has been suggested that gPDC, when accompanied by a $Z$-score pre-normalization of the time series, yields correct connectivities even for very short data samples of series with different scaling (Baccala et al. 2007). Indeed in the example of three oscillators we find that the lack of connections is correctly detected after replacing PDC with gPDC (Fig. 2). For an example of a false negative PDC, corrected by gPDC, see Baccala et al. (2007).

In our recordings the mPFC LFP signals have much lower variance than that of the hippocampal ones. This led to the application in this study of gPDC on the recordings, instead of the original PDC. This is the first study to apply gPDC on neurophysiological data.

\section{Data pre-processing and numerical implementation}

Partial directed coherence and gPDC should only be applied on stationary data. LFPs usually provide locally (almost) stationary series when examined in small segments of only a few seconds (Bernasconi and König 1999; Ding et al. 2000). As proposed by Ding et al. (2000), all LFP recording epochs were split in 10-s segments with $80 \%$ overlap, and each segment was analyzed separately. Smaller segments of 2 and $5 \mathrm{~s}$ duration were also implemented but, although yielding qualitatively similar connectivity features, their short data length resulted in poor gPDC estimation and very high critical levels.

In contrast, the binary spike trains are non-stationarity and need to be transformed into continuous stationary time series. Two methods were applied here: the spike trains (i) were substituted with their corresponding firing rates, or (ii) transformed into a continuous function through the kernel convolution method of the French-Holden algorithm (French and Holden 1971). Both methods have been used before in neurophysiological studies with PDC (Sameshima and Baccalá 
Fig. 3 The French-Holden algorithm applied on a series of spikes (top) with Nyquist frequency set to $10 \mathrm{~Hz}$ (middle) and $100 \mathrm{~Hz}$ (bottom)
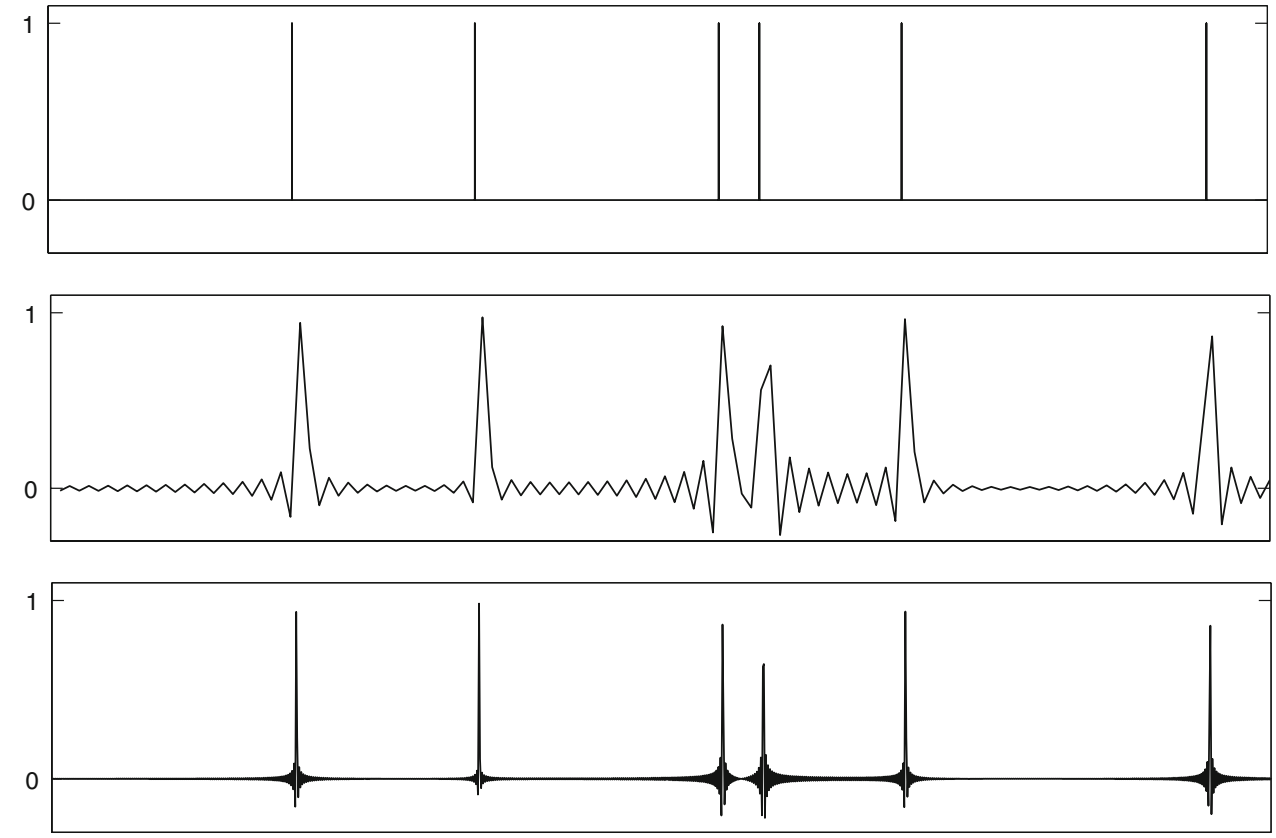

1999; Fanselow et al. 2001; Yang et al. 2005; Huang et al. 2006; Wang et al. 2007, 2008).

For the firing rate calculation, spike data were binned by $10 \mathrm{~ms}$ bins, overlapping by $50 \%$. This choice of bin length and overlap was to ensure the bins were large enough for the resulting firing rates not to be completely binary, while not losing much information by grouping the spikes. The main drawback of this method is that the information of the precise spike timings is lost.

The French-Holden $(\mathrm{F}-\mathrm{H})$ algorithm provides an alternative approach by transforming the spike point process into a continuous signal while retaining the spiking times. This is done by convolving each spike with the infinite-length kernel:

$S(t)=\frac{\sin \left(2 \pi f_{\mathrm{N}}\left(t-t_{i}\right)\right)}{2 \pi f_{\mathrm{N}}\left(t-t_{i}\right)}$

where $t_{i}$ is the timing of the specific spike and $f_{\mathrm{N}}$ is the Nyquist frequency (Marple 1987), the maximum frequency for which there will be no aliasing distortions in the final signal. The resulting infinite and continuous function is then truncated by the exact duration of the train and sampled at rate $2 f_{\mathrm{N}}$. The output is a continuous function whose magnitude represents an estimate of the instantaneous firing rate of the spike train (Paterka et al. 1978). The choice of the $f_{\mathrm{N}}$ parameter affects the shape of the final signal and the accuracy of the spike times, as can be seen in Fig. 3. In this study, the maximum frequency analyzed was $50 \mathrm{~Hz}$ and the $\mathrm{Ny}-$ quist frequency was set to $100 \mathrm{~Hz}$ to avoid significant errors in power spectral computations (Paterka et al. 1978).

Principal Component analysis was performed to group either the firing rates or the $\mathrm{F}-\mathrm{H}$ processed units of each brain region together. The first principal component from each region was implemented for the VAR modeling and subsequent PDC analysis. In most cases presented below, the first principal component contained more than $75 \%$ of the processed units' variance, rendering them good representatives of their sets of units. The only exception was the third case study where the percentage dropped to approximately $45 \%$ for the lateral hippocampal neurons. Each recording epoch was split into segments of 10 s duration with $90 \%$ overlap.

All LFP time series from each segment were normalized into $Z$-scores before further analysis. The single-unit time series had only their mean values removed, since the segments' variances were often too small to be used for normalization. VAR modeling and gPDC analysis were performed for each segment separately. Unfortunately, the very low firing of neurons under anesthesia, especially those in the mPFC, led in most occasions to very few non-zero values in the firing rates time series, even after KA administration, rendering these series non-stationary. In fact, many segments had completely zero firing rates, making it impossible to perform VAR analysis on them. Moreover, the very sparse firing led to many highly non-stationary (but non-zero) segments in the F-H processed cases as well. All the non-stationary segments of both firing rates and $\mathrm{F}-\mathrm{H}$ processed spikes yielded VAR models that did not satisfy the stability criterion (2) and their gPDC results were set to zero.

One parameter that needs to be set before VAR modeling takes place is the model order $p$ in (1). To perform a consistent analysis between all the data segments and be able to make rigorous comparisons between PDC results, the same model order should be set for all VAR models, as it represents the delay of information transfer between the recorded brain regions. This is not a straightforward choice, as different data 
sets usually require different orders for the VAR model to accurately capture the data characteristics. The model order differences are potentially even more substantial between different types of data, as in our case. Even though they correspond to the same experiment, each of the three types of neurophysiological data examined here carries a different type of information and the corresponding data have different form.

Both the Akaike and Bayesian Information criteria were applied. In the LFP cases they failed to yield an optimal order as they kept decreasing with increasing order. This is a typical situation for EEG and LFP data (Jansen 1991; Ding et al. 2000). It is customary to select a model order manually so that the power spectrum of the modeled data closely follows that of the original data (Brovelli et al. 2004; Gourévitch et al. 2006; Schelter et al. 2006b). For the spike train data, pre-processed either way, both selection criteria indicated different optimal orders that were strongly fluctuating between segments. For the LFPs a model order of 100 sample points was set, corresponding to $100 \mathrm{~ms}$ of history since the sampling rate was $1 \mathrm{KHz}$. This order yielded on average a very good fit of the model spectrum and the data spectra (although it naturally varied between different segments and different recordings). For the spike data, a delay of $100 \mathrm{~ms}$ corresponded to a model order of 20 data points for both firing rates and F-H
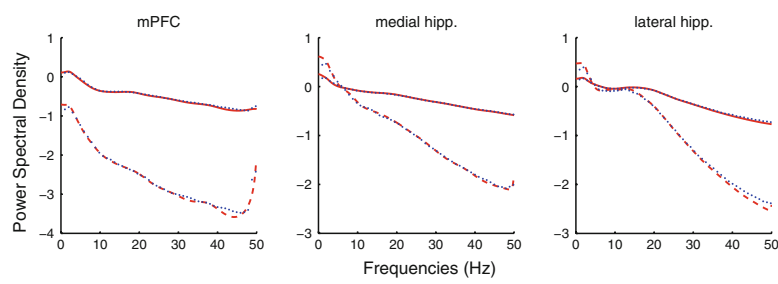

mPFC $\rightarrow$ Medial Hippocampus
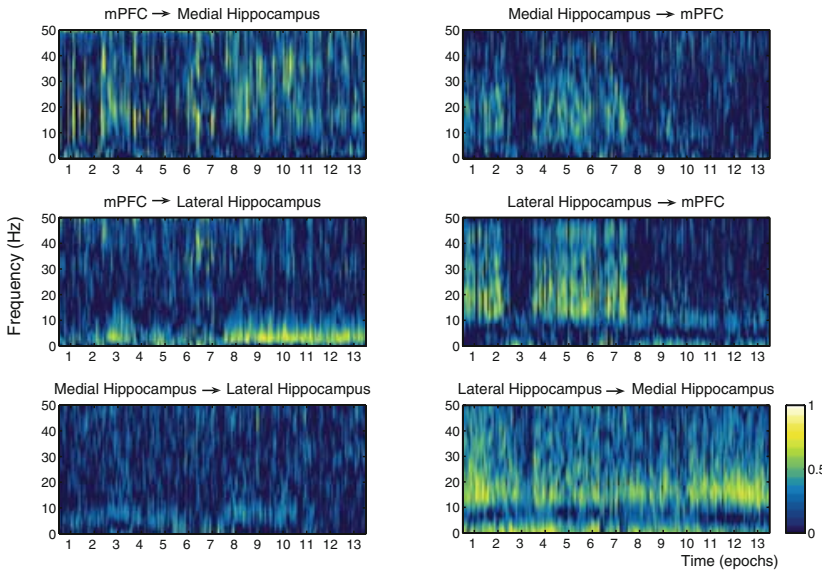

Fig. 4 Results for LFPs from Cases 1 (left column) and 2 (right column). Top Average power spectral densities of the VAR models, over the first four (basal) epochs (dashed lines) and over the last four epochs, when KA is expected to have taken effect (solid lines). LFP spectra are shown in dotted lines. Each panel represents one of the three brain regions (from left $\mathrm{mPFC}$, medial hippocampus, lateral hippocampus). processed trains. This order appeared to be close to optimal as by visual inspection, it was found that significantly increasing or decreasing $p$ diminished the fit between VAR and data spectra. The power spectra of the original data and those of the VAR models are presented for each case study in the following section as representations of the fit of the VAR models to the original data.

All data pre-processing, parameters set up, VAR modeling, PDC analysis and plots were performed through a custom-built toolbox in MATLAB, containing (modified) algorithms from the ARFIT (Neumaier and Schneider 2001; Schneider and Neumaier 2001) (http://www.gps. caltech.edu/tapio/arfit/), Biosig (http://biosig.sourceforge. net/documentation.html) and Neurospec 20 (http://www. neurospec.org/) numerical toolboxes. In particular, for the computation of the VAR models, the ARFIT algorithm was applied (Neumaier and Schneider 2001).

\section{Results}

In Figs. 4 and 6 the results from the VAR modeling and gPDC analysis on LFP data are presented for all studied cases individually, referred to as Cases 1-4. A single plot of the gPDC results averaged over all case studies was avoided so that indi-
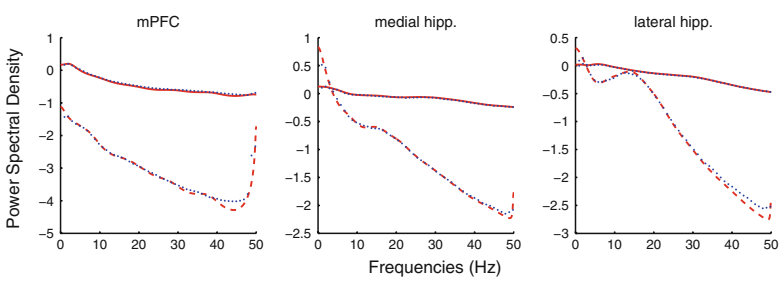

mPFC $\rightarrow$ Medial Hippocampus

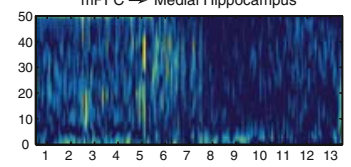

Medial Hippocampus $\rightarrow$ mPFC
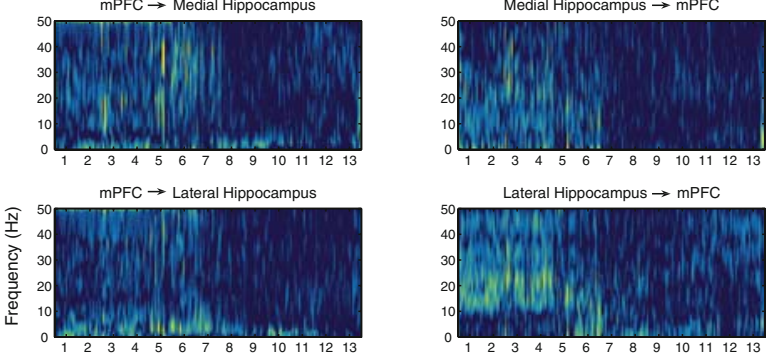

Lateral Hippocampus $\rightarrow$ mPFC
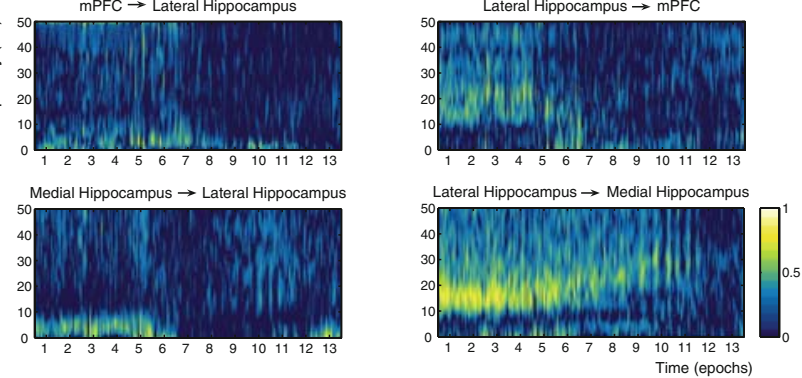

Bottom $\mathrm{gPDC}$ results between all pairs of the three brain regions. Opposite directionalities are shown next to each other. False positive gPDC entries, according to $95 \%$ critical value (11) have been set to zero. The peaks at $50 \mathrm{~Hz}$ in some panels in this (and following figures) represent $50 \mathrm{~Hz}$ mains noise artefacts 
(a)

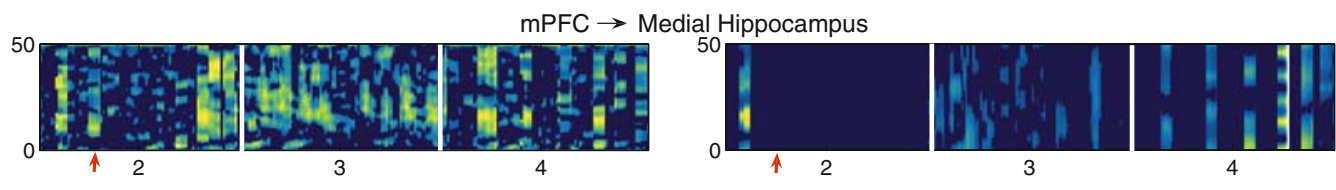

$\mathrm{mPFC} \rightarrow$ Lateral Hippocampus
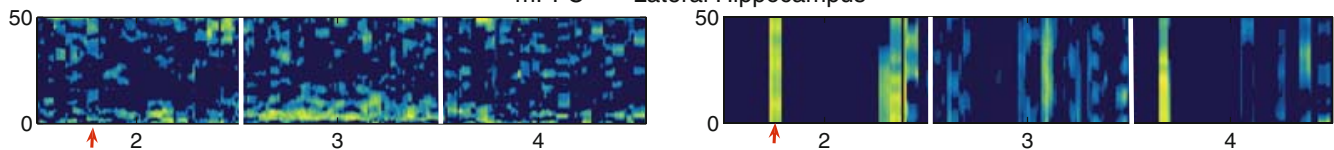

Medial Hippocampus $\rightarrow$ mPFC
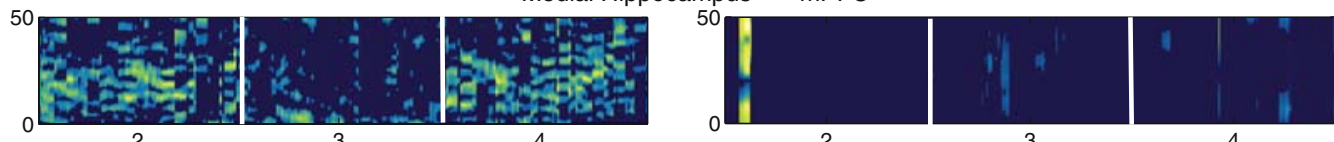

Lateral Hippocampus $\rightarrow$ mPFC

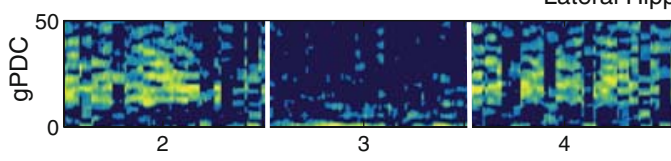

(b)

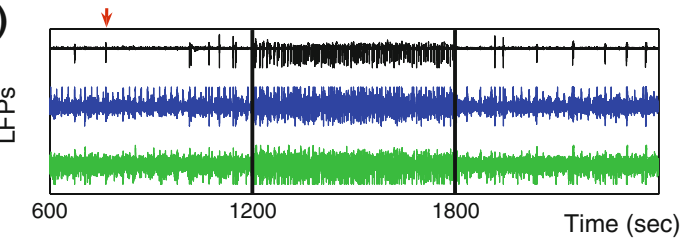

Fig. 5 a gPDC results between the mPFC and the hippocampus of Case 1 during the second to fourth recording epochs, plotted in similar fashion as in Fig. 4. Left row displays gPDC from LFP recordings while the right one displays gPDC from $\mathrm{F}-\mathrm{H}$ processed spike data. White lines indicate the limits between the epochs. b LFP recordings from the mPFC (top), medial (middle) and lateral hippocampus (bottom) during

vidual differences and similarities in neural network dynamics could be examined. For each one, the gPDC results are presented along with the spectral comparison between the original data and the VAR model. This comparison is done separately for spectra averaged over the first four recording epochs (344 segments in total) of basal recordings and for spectra averaged over the last four epochs during which KA is expected to have taken full effect. The averaged power spectra are plotted in the top panel row in each case, separately for each of the three brain regions under study.

In Case 1 (Fig. 4, left column), gPDC indicates a bidirectional functional connection between the $\mathrm{mPFC}$ and the hippocampus. Information appears to be flowing continuously from the medial hippocampus to the mPFC, mostly in the beta frequency band $(10-30 \mathrm{~Hz})$, with the $\mathrm{mPFC}$ responding mainly during short bursts of activity where gPDC spans most of the frequency range. Also the lateral hippocampus sends information to $\mathrm{mPFC}$ at frequencies $>10 \mathrm{~Hz}$, again peaking roughly around the beta band. $\mathrm{mPFC}$ seems to be responding to lateral hippocampus mostly in the lower frequencies around the $0-10 \mathrm{~Hz}$ frequency band. gPDC also revealed a strong intra-hippocampal connection from the lat- the same epochs. The intervals of no recordings between the epochs have been removed. Black lines indicate the limits between epochs and time axis displays the starting time of each epoch (180 s duration). The arrows indicate the second UP state in the MPFC signal during epoch 2 and the mPFC-to-hippocampus PDC results corresponding to that segment. These results are discussed in the text

eral to medial hippocampus, mostly concentrated on the beta $(10-30 \mathrm{~Hz})$ frequency band, with a gPDC maximum around $\sim 15 \mathrm{~Hz}$. Causality in the opposite direction is very low, peaking in the theta band $(4-10 \mathrm{~Hz})$. KA started taking effect after the 7th epoch but did not disrupt the strong intra-hippocampal connection, while its effects were more evident in the cortico-hippocampal ones. Information flow from hippocampus to cortex was halted and reversed, with strong flow in the opposite direction, from mPFC to both medial hippocampus (covering most frequencies) and lateral (localized in the $0-10 \mathrm{~Hz}$ band).

One distinctive feature of the basal activity epochs in Case 1 is a large gPDC decrease from hippocampus to $\mathrm{mPFC}$ during the third epoch with a simultaneous increase in the opposite direction, identical to the connectivity during the KA-induced enhanced-activity epochs. This reversal of directionality coincided with a period of continuous oscillatory transitions between active and quiet states in the cortex. Figure 5a displays gPDC between $\mathrm{mPFC}$ and hippocampus during epochs 2 to 4 . Results from LFP analysis are shown on the left row, while the right one contains the corresponding results from $\mathrm{F}-\mathrm{H}$ processed spike trains. Due to the very 

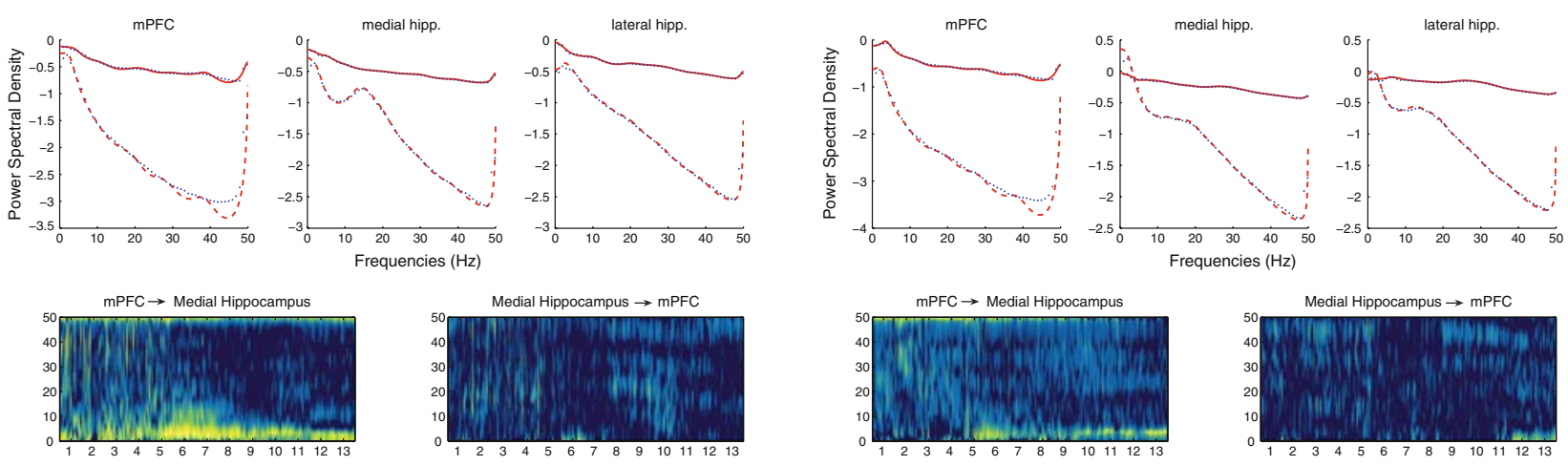

mPFC $\rightarrow$ Lateral Hippocampus

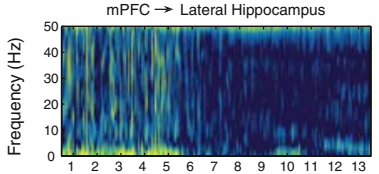

Lateral Hippocampus $\rightarrow$ mPFC

$\mathrm{mPFC} \rightarrow$ Lateral Hippocampus

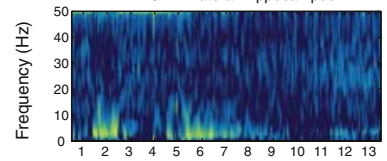

Lateral Hippocampus $\rightarrow$ mPFC

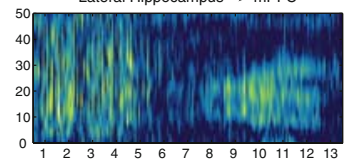

Medial Hippocampus $\rightarrow$ Lateral Hippocampus
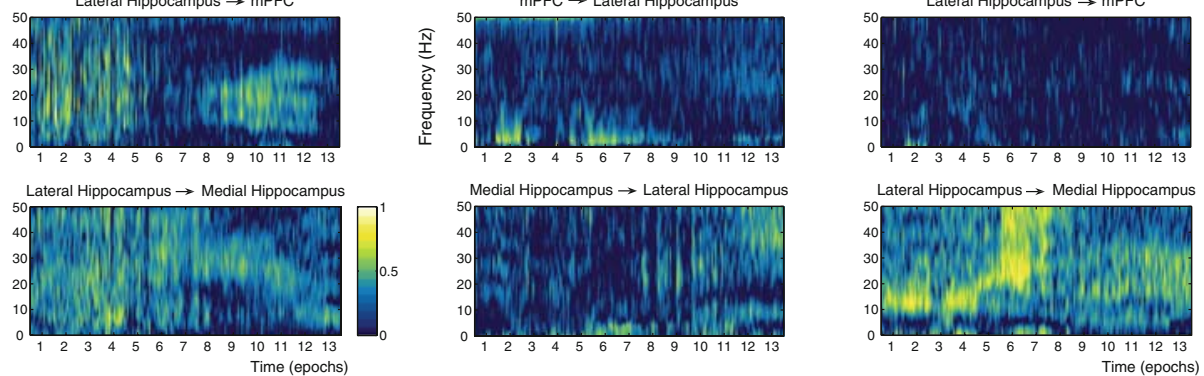

Lateral Hippocampus $\rightarrow$ Medial Hippocampus
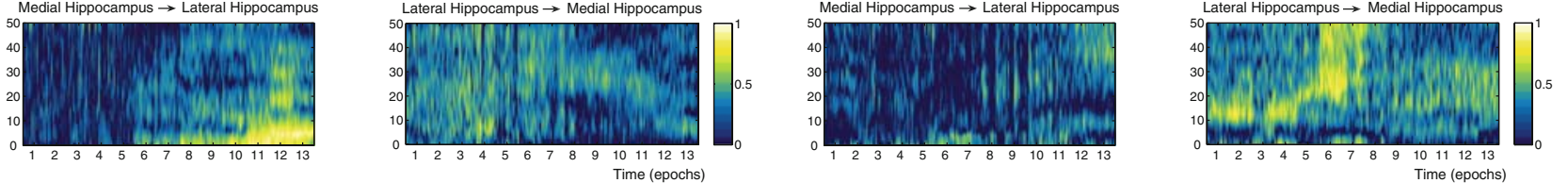

Fig. 6 Same set of plots as in Fig. 4 for the third (left) and fourth (right) case studies

sparse firing, especially in the $\mathrm{mPFC}$ area, many segments gave unstable VAR models. gPDC from these segments was set to zero. For the same reason, VAR modeling of the firing rates was not possible since most segments yielded only zero values in the firing rate time series. Nevertheless, some segments gave significant non-zero gPDC. Figure 5b displays the LFP recordings from the same three epochs.

Clearly, the continuous hippocampus-to-mPFC information flow is interrupted and reversed only during short bursts of activity in mPFC (UP states). These bursts gave rise to high gPDC from mPFC towards mostly the medial hippocampal area. This reversed connectivity was sustained throughout the third epoch where mPFC exhibited a continuous oscillation between UP states and relaxation (DOWN states), sending information to hippocampus. Spike trains reveal the same scenario. Intuitively, significant gPDC results appeared only during the mPFC UP states when action potentials were actually produced. Again, it appears that those states coincided with information flow towards the hippocampus. Consequently, the third epoch gave rise to continuous high mPFCto-hippocampus gPDC. It is noteworthy that in some cases, results from spikes and LFPs reveal different hippocampal areas as targets of mPFC information flow. For example, during the second UP state in epoch 2 (arrow in Fig. 5b), spike train analysis indicated information flow only towards the lateral hippocampus, while gPDC from LFPs suggests mainly flow towards the medial (arrows in Fig. 5a).

In Case 2 (Fig. 4 right column), gPDC yielded similar basal connectivity profile as in Case 1 . The cortico- hippocampal connection appears reciprocal, with the lateral hippocampus sending no information below approximately $10 \mathrm{~Hz}$ and the mPFC sending information back predominantly below this frequency boundary. Again most of the intra-hippocampal information flow is found in the lateralto-medial pathway, on the beta band, with flow below this range going only in the opposite direction, towards the lateral hippocampus. The disruptive effect of KA in the cortico-hippocampal connections is also evident here but this time with no apparent reversal of flow towards the hippocampus. This time KA also disrupted the intra-hippocampal connectivity as well, as there is a gradual drop of gPDC in the lateralto-medial pathway and a dramatic reduction in the opposite direction. This reduction is followed by a small increase on higher frequencies suggesting a partial reversal of intra-hippocampal connectivity.

Case 3 (Fig. 6, left column) presents a similar strong reciprocal mPFC-lateral hippocampal connection as before. Here, the medial hippocampus-to-mPFC connection appears much less prominent, while the high gPDC values in the mPFC-tohippocampus connections, present mostly in short bursts of activity, are more concentrated on low frequencies. Intra-hippocampal connections are again directed from the lateral to the medial although the strong peak on the beta band is now absent. KA took effect sooner in Case 3 (6th epoch compared to 8 th and 7 th in the two previous cases). Again it disrupted information flow from hippocampus to mPFC, but only for a transient period. After epoch 9 the basal connectivity is partly restored. Also, here the mPFC-to-medial hippocampus 
Fig. 7 Results for the first principal components of $\mathrm{F}-\mathrm{H}$ processed spike trains in Case 4. The sets of plots are similar to the ones in Figs. 4 and 6. Only the power spectra of the last four epochs are displayed as the basal epochs contained too many unstable VAR models. We note that gPDC analysis on the last seven epochs of firing-rates processed spike trains gave identical results as the ones presented here
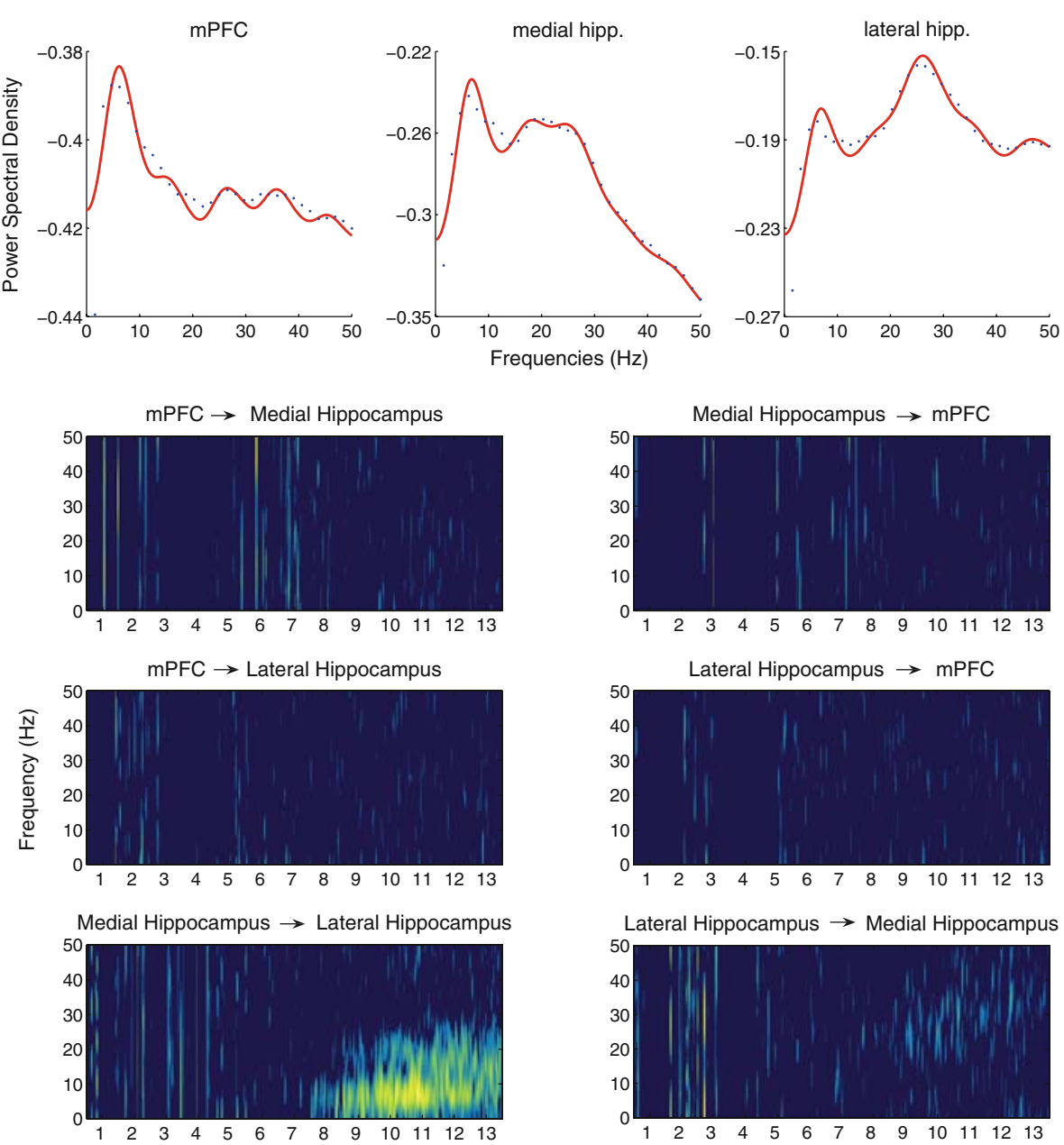

connection was greatly enhanced in the low frequency band $(0-10 \mathrm{~Hz})$, while the one to the lateral was interrupted by KA. Finally, KA action also resulted in a partial reversal of the intra-hippocampal connectivity, with gPDC presenting a substantial drop of the medial-to-lateral hippocampus connection strength and a simultaneous rapid increase in the opposite direction, most prominently in the low frequency band.

In Case 4 (Fig. 6, right column), the hippocampus-tomPFC connections are almost absent. From the mPFC, information flow is directed mostly towards the medial hippocampus, with only a transient increase towards the lateral, corresponding to a period of similar UP/DOWNtransitions in mPFC-activity as epoch 3 in Case 1. The most distinctive characteristic in the intra-hippocampal connectivity is again the very strong gPDC in the lateral-to-medial pathway on a frequency range around the beta band where gPDC is maximal while in the theta band it is almost absent. This strong connectivity is again partially reversed under KA activity, while the mPFC-to-medial hippocampus connection is enhanced on the low frequencies as in Case 3.
Case 4 exhibited a firing rate after KA administration which was high enough to allow gPDC analysis to be performed on spike data. However, the very sparse firing during the initial basal epochs allowed VAR modeling of the firing rates to be performed only on the last seven epochs. It also resulted in many segments with unstable VAR models for the F-H processed spikes. In Fig. 7, gPDC results from the $\mathrm{F}-\mathrm{H}$ processed spikes are displayed in a similar fashion as for the LFP results. Power spectra comparisons are performed only for the KA epochs where unstable VAR segments were sparse. Results from the firing rates are not shown as they were practically identical with the last seven epochs in Fig. 7. The basal epochs exhibit no specific sustained connectivity. The most distinctive characteristic is the pronounced increase in medial-to-lateral hippocampal connectivity after the KA administration, sustained until the end of the recordings. This increase coincides temporally with the gPDC increase observed in the corresponding LFP results. Although it does not verify the reversal in hippocampal connectivity observed through LFPs, it supports the KA-induced increase of information flow from medial to lateral hippocampus. 


\section{Discussion}

A significant challenge in neuroscience lies in determining how complex interactions between discrete populations of neurones elicit functional output. Partial directed coherence analysis was applied to LFP and unit activity recorded at mPFC and medial (CA1) and lateral (CA3) hippocampal recording sites, to address interactions between these regions. A variation of the original PDC (Sameshima and Baccalá 1999; Baccalá and Sameshima 2001), notably generalized PDC (Baccala et al. 2007), was used in order to avoid distorted connectivity results due to different scaling of the data in cortex and hippocampus. Spike train data from each brain region were replaced by their firing rate or a continuous function generated through the $\mathrm{F}-\mathrm{H}$ algorithm and grouped using Principal Component analysis.

Connectivity results were consistent between LFP recordings during basal recording conditions. Within the hippocampus, the predominant connectivity under isoflurane anesthesia was clearly from lateral towards medial, concentrated in the beta $(10-30 \mathrm{~Hz})$ frequency band, peaking strongly around $15 \mathrm{~Hz}$. Usually, little information was sent in the opposite direction, and then mostly in the theta (4$10 \mathrm{~Hz}$ ) frequency band. This lateral-to-medial directionality was consistent with the established neuroanatomy of the hippocampus, i.e., glutamatergic pyramidal cells located in CA3 projecting, via Schaffer collaterals, to pyramidal cells in CA1 (reviewed by Amaral and Witter 1989).

Analysis of LFP activity further revealed a reciprocal flow of information between the mPFC and hippocampus. In most cases, the hippocampus showed an almost continuous information flow towards the mPFC, with the lateral hippocampus-to-mPFC gPDC peaking predominately between 10 and $30 \mathrm{~Hz}$. In the opposite direction, the mPFC-to-hippocampal information flow was less strong during periods of $\mathrm{mPFC}$ inactivity (corresponding with the "DOWN-state"), but interestingly peaked during short bursts of mPFC ("UP-state") activity. This suggests that the hippocampus feeds the mPFC, while the latter is silent, in an almost-continuous fashion with signals at frequencies $>10 \mathrm{~Hz}$, with the $\mathrm{mPFC}$ responding in bursts towards both the medial (over most of the frequency range) and the lateral hippocampus (mostly at frequencies $<10 \mathrm{~Hz}$ ).

The hippocampus forms multiple connections with other structures in the brain, including the mPFC. Specifically, the hippocampus (ventral CA1) projects to $\mathrm{mPFC}$, including the medial orbital area, prelimbic and infralimbic cortices, while no projections from CA2, CA3, or dentate gyrus to $\mathrm{mPFC}$ exist (Goldman-Rakic et al. 1984; Ferino et al. 1987; Jay et al. 1989; Sesack et al. 1989; Jay and Witter 1991; Carr and Sesack 1996). The present recordings were made in the dorsal hippocampus and functional intrinsic connectivity within the hippocampus requires addressing in the future. This

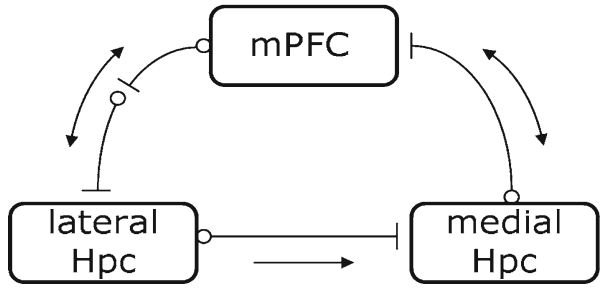

Fig. 8 Simplified schematic representation of the information flow, under basal conditions, between the structures recorded in the present study. Schaffer collaterals project from lateral (CA3) to the medial (CA1) hippocampal area (Hpc) and monosynaptic projections have been reported from medial hippocampus (CA1) to the mPFC. No monosynaptic pathways have been observed from $\mathrm{mPFC}$ back to the hippocampus, but it is possible that other areas can act as relay structures (see text). The arrows represent the directionality as assessed by gPDC

CA1-mPFC projection is primarily unidirectional, given that no direct return projections have been identified (GoldmanRakic et al. 1984; Room et al. 1985; Sesack et al. 1989; Hurley et al. 1991; Takagishi and Chiba 1991; Buchanan et al. 1994). The monosynaptic hippocampal-to-mPFC pathway innervates both pyramidal cells and interneurons in the rat (Ferino et al. 1987; Laroche et al. 1990; Jay et al. 1995; Tierney et al. 2004), and has been implicated in memory acquisition and consolidation, specifically for working spatial memory (reviewed in Goldman-Rakic 1995) (Fig. 8).

Interestingly, the observed mPFC-to-hippocampus directionality appears inconsistent with an unidirectional monosynaptic projection from hippocampus to mPFC. This observation may be explained by the participation of a "relay" structure, notably the nucleus reuniens of the thalamus, a structure that receives strong input from prelimbic and infralimbic cortices of the mPFC and projects to the hippocampus (Vertes 2006; Vertes et al. 2007).

Recent electrophysiological studies (reviewed by Tononi et al. 2006), suggest that the neocortical slow oscillation (Steriade et al. 1993; Steriade 2006) which engages neurons in prefrontal and other cortical regions into synchronous transitions between depolarized (UP-) and hyperpolarized (DOWN-) states during deep sleep and anesthesia, influences activity in hippocampal cells even though such activity lacks the neocortical bimodality of the slow oscillation (Isomura et al. 2006). Specifically, it has been suggested that cortical UP-states result in increased activity of dentate and most CA1 neurons, as well as a higher probability of ripple events in the hippocampus (Isomura et al. 2006). The delay between this cortical influence and its effect on hippocampus is in the order of tens of milliseconds (Molle et al. 2006). The high model order that was applied during the present study contains such delays, and the gPDC results presented in Fig. 5 (epoch 3) seem to support this notion, implying that during slow oscillation-type cortical activity, information flows mainly from mPFC to hippocampus. Further, these results 
suggest that this information flow appears generally during the cortical UP-state, even isolated ones.

It can be argued that the clear increase in mPFC-to-medial hippocampus gPDC observed during these UP-states is a numerical artifact produced by poor VAR modeling, resulting from the non-stationarity that an UP-state introduces in a modeled LFP segment. Nevertheless, the absence of similarly strong gPDC increase in the corresponding mPFC-to-lateral hippocampus panels supports the validity of our results.

In this study, kainic acid administration resulted in a variety of effects on connectivity profiles, yet two major effects occurred in most case studies: (i) KA caused a partial disruption of connectivity between the mPFC and hippocampus, in both directions and (ii) following KA there was a weakening in the lateral-to-medial connection, often accompanied with a strong increase of the opposite pathway, leading to a gradual and partial reversal of connectivity in the hippocampus.

The use of KA is well established as a model of status epilepticus (SE) and spontaneous seizures in the rat, and the hippocampus is particularly susceptible to pharmacological manipulation with KA (Lothman et al. 1981; Nadler 1981; Ben-Ari 1985; Buckmaster and Dudek 1999). KA receptors are broadly distributed in the hippocampus, presynaptically regulating glutamate release at mossy fiber-CA3 synapses (Schmitz et al. 2001), and GABA release between interneurones (Mulle et al. 2000; Cossart et al. 2001) and at interneurone-pyramidal cell synapses (Clarke et al. 1997; RodríguezMoreno et al. 1997; Maingret et al. 2005). The mechanisms by which SE and spontaneous seizures are generated in animal models with KA are not fully understood. Activation of high affinity KA receptors (densely expressed at mossy fiber synapses), coupled with additional excitation (leading to synchronization) provided by recurrent collateral projections between CA3 pyramidal neurones is proposed to underlie epileptiform activity generated as a result of KA administration. This occurs in spite of increased GABA-mediated inhibition (Ben-Ari and Cossart 2000). Alternatively, a removal of inhibition at interneurone-pyramidal cell synapses may also be involved (Clarke et al. 1997; Rodríguez-Moreno et al. 1997; Maingret et al. 2005). Epileptiform activity subsequently propagates from CA 3 to CA1, and then, via the subiculum, to other limbic structures (Ben-Ari and Cossart 2000)

Our results confirm the particular susceptibility of the hippocampus to KA. The decline in lateral-to-medial hippocampal information flow, and the subsequent reversal of directionality, exhibited by some rats administered KA in these experiments is intriguing, in light of both the described anatomy of the hippocampus, and the suggested mechanisms for KA-induced limbic seizure. The perforant path does not represent the sole pathway within the hippocampus (e.g., the entorhinal cortex projects directly to CA1 via the temporo-ammonic pathway); however, reverse projections from
CA1 to CA3 have not been reported. Furthermore the susceptibility of CA3 mossy fibers to KA does not fit with medial-to-lateral directionality. One caveat is the influence of anesthesia on KA-evoked activity which may underlie the hippocampal directionality observed in this study. In addition, different sub-populations of neurones may have been recorded because of variations in the precise array-placement between experiments.

Alterations to functional connectivity have implications for memory processing in epilepsy. Temporal lobe seizures are associated with functional deficits, including working memory (Schubert et al. 2005), for which the hippocampus and mPFC are both important (Goldman-Rakic 1995).

Our results further indicate that applying PDC on spike trains can be problematic due to their highly non-stationary nature. Pre-processing of the unit data is necessary in order to generate a stationary series. While the effects of such processing may not always be clear they should be taken into account in subsequent analysis. Both of the pre-processing techniques applied here yielded similar results as parameters (i.e., bin length, overlap) were chosen so that the resulting firing rate did not deviate much from the instantaneous firing rate given through the $\mathrm{F}-\mathrm{H}$ algorithm. In general, processing parameters should be chosen carefully, and according to the specific case under study. For example, grouping the spikes into large bins leads to a loss of information about the firing patterns of the neuron. Conversely, small bin sizes may be inadequate for a low-firing neuron. In this particular study, anesthesia resulted in low firing rates. Thus, spike train gPDC analysis did not reveal any significant connectivity, with the exception of Case 4. Nevertheless, in this recording, spike train gPDC did parallel elements of the connectivity revealed by corresponding analysis on LFPs, namely the increase in medial-to-lateral hippocampus information flow under KA. The same conclusion can be drawn from the analysis of the three basal epochs of Case 1 presented in Fig. 5. Despite the very low firing of $\mathrm{mPFC}$, spike train analysis did support the mPFC-to-hippocampus connectivity during the mPFC UP-states. Thus gPDC analysis of spike trains can be a useful complementary tool along with analysis of LFPs.

PDC represents a novel technique for assessing interactions between brain regions and revealing their directionality. Its variation, gPDC, is a powerful tool which, combined with data normalization, can overcome distortions caused by differences in the electrophysiological signal scaling. Nevertheless, caution is necessary when applying such statistical tools in the analysis of neurophysiological data as inappropriate use can lead to false conclusions. There are many issues such as the scaling and stationarity of time series data and PDC model order that need to be taken into account when constructing the VAR models, which is the most crucial part of this analysis approach. 
Acknowledgments Special thanks to Prof. Frank Ball and Dr. Theodore Kypraios for their help in issues of statistical analysis. This study was funded by the Marie Curie Early Stage Research Fellowships.

Open Access This article is distributed under the terms of the Creative Commons Attribution Noncommercial License which permits any noncommercial use, distribution, and reproduction in any medium, provided the original author(s) and source are credited.

\section{References}

Akaike H (1974) A new look at the statistical model identification. IEEE Trans Autom Control 19(6):716-723

Amaral D, Witter M (1989) The three-dimensional organization of the hippocampal formation: a review of anatomical data. Neuroscience 31(3):571-591

Astolfi L, Cincotti F, Mattia D, Marciani M, Baccala L, de Vico Fallani F, Salinari S, Ursino M, Zavaglia M, Ding L et al (2007) Comparison of different cortical connectivity estimators for high-resolution EEG recordings. Hum Brain Mapp 28(2):143

Baccalá L, Sameshima K (2001) Partial directed coherence: a new concept in neural structure determination. Biol Cybern 84(6):463-474

Baccala L, Takahashi D, Sameshima K (2007) Generalized partial directed coherence. In: 15th international conference on digital signal processing, pp 163-166

Ben-Ari Y (1985) Limbic seizure and brain damage produced by kainic acid: mechanisms and relevance to human temporal lobe epilepsy. Neuroscience 14(2):375-403

Ben-Ari Y, Cossart R (2000) Kainate, a double agent that generates seizures: two decades of progress. Trends Neurosci 23(11):580-587

Bernasconi C, König P (1999) On the directionality of cortical interactions studied by structural analysis of electrophysiological recordings. Biol Cybern 81(3):199-210

Brovelli A, Ding M, Ledberg A, Chen Y, Nakamura R, Bressler S (2004) Beta oscillations in a large-scale sensorimotor cortical network: directional influences revealed by Granger causality. Proc Natl Acad Sci 101(26):9849-9854

Buchanan S, Thompson R, Maxwell B, Powell D (1994) Efferent connections of the medial prefrontal cortex in the rabbit. Exp Brain Res 79(2):469-483

Buckmaster P, Dudek F (1999) In vivo intracellular analysis of granule cell axon reorganization in epileptic rats. J Neurophysiol 81(2):712-721

Cadotte A, DeMarse T, He P, Ding M (2008) Causal measures of structure and plasticity in simulated and living neural networks. PLoS ONE 3(10):1-14

Carr D, Sesack S (1996) Hippocampal afferents to the rat prefrontal cortex: synaptic targets and relation to dopamine terminals. J Comp Neurol 369(1):1-15

Clarke V, Ballyk B, Hoo K, Mandelzys A, Pellizzari A, Bath C, Thomas J, Sharpe E, Davies C, Ornstein P et al (1997) A GluR5 kainate receptor that regulates inhibitory synaptic transmission in the hippocampus. Nature 389:599-603

Coomber B, O'Donoghue M, Mason R (2008) Inhibition of endocannabinoid metabolism attenuates enhanced hippocampal neuronal activity induced by kainic acid. Synapse 62(10):746-755

Cossart R, Tyzio R, Dinocourt C, Esclapez M, Hirsch J, Ben-Ari Y, Bernard C (2001) Presynaptic kainate receptors that enhance the release of GABA on CA1 hippocampal interneurons. Neuron 29(2):497-508

Ding M, Bressler S, Yang W, Liang H (2000) Short-window spectral analysis of cortical event-related potentials by adaptive multivariate autoregressive modeling: data preprocessing, model validation, and variability assessment. Biol Cybern 83(1):35-45
Fanselow E, Sameshima K, Baccala L, Nicolelis M (2001) Thalamic bursting in rats during different awake behavioral states. Proc Natl Acad Sci 98(26):15330-15335

Ferino F, Thierry A, Glowinski J (1987) Anatomical and electrophysiological evidence for a direct projection from Ammon's horn to the medial prefrontal cortex in the rat. Exp Brain Res 65(2): $421-426$

Frank L, Brown E, Wilson M (2001) A comparison of the firing properties of putative excitatory and inhibitory neurons from CA1 and the entorhinal cortex. J Neurophysiol 86(4):2029-2040

French A, Holden A (1971) Alias-free sampling of neuronal spike trains. Biol Cybern 8(5):165-171

Goldman-Rakic P (1995) Cellular basis of working memory. Neuron 14(3):477-485

Goldman-Rakic P, Selemon L, Schwartz M (1984) Dual pathways connecting the dorsolateral prefrontal cortex with the hippocampal formation and parahippocampal cortex in the rhesus monkey. Neuroscience 12(3):719-743

Gourévitch B, Bouquin-Jeannès R, Faucon G (2006) Linear and nonlinear causality between signals: methods, examples and neurophysiological applications. Biol Cybern 95(4):349-369

Granger CWJ (1980) Investigating causal relations by econometric models and cross-spectral methods. Econometrica 37:424-438

Halliday D, Rosenberg J, Amjad A, Breeze P, Conway B, Farmer $\mathrm{S}$ (1995) A framework for the analysis of mixed time series/point process data - theory and application to the study of physiological tremor, single motor unit discharges and electromyograms. Prog Biophys Mol Biol 64(2-3):237-278

Hong L, Mubarak W, Sunami Y, Murakami S, Fuyama Y, Ohtsuka A, Murakami T (2000) Enhanced visualization of weak colloidal iron signals with Bodians protein silver for demonstration of perineuronal nets of proteoglycans in the central nervous system. Arch Histol Cytol 63(5):459-465

Huang J, Chang J, Woodward D, Baccalá L, Han J, Wang J, Luo F (2006) Dynamic neuronal responses in cortical and thalamic areas during different phases of formalin test in rats. Exp Neurol 200(1):124-134

Hurley K, Herbert H, Moga M, Saper C (1991) Efferent projections of the infralimbic cortex of the rat. J Comp Neurol 308(2):249-276

Isomura Y, Sirota A, Özen S, Montgomery S, Mizuseki K, Henze D, Buzsáki G (2006) Integration and segregation of activity in entorhinal-hippocampal subregions by neocortical slow oscillations. Neuron 52(5):871-882

Jansen B (1991) Time series analysis by means of linear modelling. Tech Behav Neural Sci 5:157-180

Jay T, Witter M (1991) Distribution of hippocampal CA1 and subicular efferents in the prefrontal cortex of the rat studied by means of anterograde transport of Phaseolus vulgaris-leucoagglutinin. J Comp Neurol 313(4):574-586

Jay T, Glowinski J, Thierry A (1989) Selectivity of the hippocampal projection to the prelimbic area of the prefrontal cortex in the rat. Brain Res 505(2):337-340

Jay T, Burette F, Laroche S (1995) NMDA receptor-dependent longterm potentiation in the hippocampal afferent fibre system to the prefrontal cortex in the rat. Eur J Neurosci 7(2):247-250

Kamiński M, Blinowska K (1991) A new method of the description of the information flow in the brain structures. Biol Cybern 65(3):203-210

Kamiński M, Ding M, Truccolo W, Bressler S (2001) Evaluating causal relations in neural systems: Granger causality, directed transfer function and statistical assessment of significance. Biol Cybern $85(2): 145-157$

Kunz T, Oliw E (2001) The selective cyclooxygenase-2 inhibitor rofecoxib reduces kainate-induced cell death in the rat hippocampus. Eur J Neurosci 13(3):569-575 
Kus R, Kaminski M, Blinowska K (2004) Determination of EEG activity propagation: pair-wise versus multichannel estimate. IEEE Trans Biomed Eng 51(9):1501-1510

Laroche S, Jay T, Thierry A (1990) Long-term potentiation in the prefrontal cortex following stimulation of the hippocampal CA1/subicular region. Neuroscience Lett 114(2):184-190

Lothman E, Collins R, Ferrendelli J (1981) Kainic acid-induced limbic seizures: electrophysiologic studies. Neurology 31(7):806-812

Lütkepohl H (2005) New introduction to multiple time series analysis. Springer, Berlin

Maingret F, Lauri S, Taira T, Isaac J (2005) Profound regulation of neonatal CA1 rat hippocampal GABAergic transmission by functionally distinct kainate receptor populations. J Physiol 567(1):131142

Marple S (1987) Digital spectral analysis. Prentice-Hall, Englewood Cliffs, NJ

Molle M, Yeshenko O, Marshall L, Sara S, Born J (2006) Hippocampal sharp wave-ripples linked to slow oscillations in rat slow-wave sleep. J Neurophysiol 96(1):62-70

Mulle C, Sailer A, Swanson G, Brana C, O'Gorman S, Bettler B, Heinemann S (2000) Subunit composition of kainate receptors in hippocampal interneurons. Neuron 28(2):475-484

Nadler J (1981) Kainic acid as a tool for the study of temporal lobe epilepsy. Life Sci 29:2031-2042

Neumaier A, Schneider T (2001) Estimation of parameters and eigenmodes of multivariate autoregressive models. ACM Trans Math Softw (TOMS) 27(1):27-57

Paterka R, Sanderson A, O'leary D (1978) Practical considerations in the implementation of the French-Holden algorithm for sampling of neuronal spike trains. IEEE Trans Biomed Eng 25:192-195

Paxinos G, Watson C (1998) The rat brain in stereotaxic coordinates, 4th edn. Academic Press, San Diego, CA

Pereda E, Quiroga R, Bhattacharya J (2005) Nonlinear multivariate analysis of neurophysiological signals. Prog Neurobiol 77(12): $1-37$

Rodríguez-Moreno A, Herreras O, Lerma J (1997) Kainate receptors presynaptically downregulate GABAergic inhibition in the rat hippocampus. Neuron 19(4):893-902

Room P, Russchen F, Groenewegen H, Lohman A (1985) Efferent connections of the prelimbic (area 32) and the infralimbic (area 25) cortices: an anterograde tracing study in the cat. J Comp Neurol 242(1):40-55

Sameshima K, Baccalá L (1999) Using partial directed coherence to describe neuronal ensemble interactions. J Neurosci Methods 94(1):93-103

Schelter B, Winterhalder M, Eichler M, Peifer M, Hellwig B, Guschlbauer B, Lücking C, Dahlhaus R, Timmer J (2006a) Testing for directed influences among neural signals using partial directed coherence. J Neurosci Methods 152(1-2):210-219

Schelter B, Winterhalder M, Hellwig B, Guschlbauer B, Lücking C, Timmer J (2006b) Direct or indirect? Graphical models for neural oscillators. J Physiol Paris 99(1):37-46

Schmitz D, Mellor J, Nicoll R (2001) Presynaptic kainate receptor mediation of frequency facilitation at hippocampal mossy fiber synapses. Science 291(5510):1972-1976

Schneider T, Neumaier A (2001) Algorithm 808: ARfit A Matlab package for the estimation of parameters and eigenmodes of multivariate autoregressive models. ACM Trans Math Softw (TOMS) 27(1):58-65

Schubert M, Siegmund H, Pape H, Albrecht D (2005) Kindling-induced changes in plasticity of the rat amygdala and hippocampus. Learn Memory 12(5):520-526
Schwarz G (1978) Estimating the dimension of a model. Ann Stat 6(2):461-464

Sesack S, Deutch A, Roth R, Bunney B (1989) Topographical organization of the efferent projections of the medial prefrontal cortex in the rat: an anterograde tract-tracing study with Phaseolus vulgaris leucoagglutinin. J Comp Neurol 290(2):213-242

Steriade M (2006) Grouping of brain rhythms in corticothalamic systems. Neuroscience 137(4):1087-1106

Steriade M, Nunez A, Amzica F (1993) A novel slow $(<1 \mathrm{~Hz})$ oscillation of neocortical neurons in vivo: depolarizing and hyperpolarizing components. J Neurosci 13(8):3252-3265

Stevenson C, Halliday D, Marsden C, Mason R (2007) Systemic administration of the benzodiazepine receptor partial inverse agonist FG-7142 disrupts corticolimbic network interactions. Synapse 61(8):646-663

Takagishi M, Chiba T (1991) Efferent projections of the infralimbic (area 25) region of the medial prefrontal cortex in the rat: an anterograde tracer PHA-L study. Brain Res 566(1-2):26-39

Takahashi D, Baccalá L (2007) Connectivity inference between neural structures via partial directed coherence. J Appl Stat 34(10):12591273

Tierney P, Degenetais E, Thierry A, Glowinski J, Gioanni Y (2004) Influence of the hippocampus on interneurons of the rat prefrontal cortex. Eur J Neurosci 20(2):514-524

Tononi G, Massimini M, Riedner B (2006) Sleepy dialogues between cortex and hippocampus: who talks to whom?Neuron 52(5):748749

Vertes R (2006) Interactions among the medial prefrontal cortex, hippocampus and midline thalamus in emotional and cognitive processing in the rat. Neuroscience 142(1):1-20

Vertes R, Hoover W, Szigeti-Buck K, Leranth C (2007) Nucleus reuniens of the midline thalamus: link between the medial prefrontal cortex and the hippocampus. Brain Res Bull 71(6):601-609

Wang J, Chang J, Woodward D, Baccala L, Han J, Luo F (2007) Corticofugal influences on thalamic neurons during nociceptive transmission in awake rats. Synapse 61(5):335-342

Wang J, Zhang H, Chang J, Woodward D, Baccalá L, Luo F (2008) Anticipation of pain enhances the nociceptive transmission and functional connectivity within pain network in rats. Mol Pain 4(1):34

Westbrook G, Lothman E (1983) Cellular and synaptic basis of kainic acid-induced hippocampal epileptiform activity. Brain Res 273(1):97-109

Winterhalder M, Schelter B, Hesse W, Schwab K, Leistritz L, Klan D, Bauer R, Timmer J, Witte H (2005) Comparison of linear signal processing techniques to infer directed interactions in multivariate neural systems. Signal Process 85(11):2137-2160

Yang H, Chang J, Woodward D, Baccalá L, Han J, Luo F (2005) Coding of peripheral electrical stimulation frequency in thalamocortical pathways. Exp Neurol 196(1):138-152 